\title{
EL INCREMENTO DEL RIESGO POR COVID-19 EN COLOMBIA A RAIIZ DEL ESTADO DE LA INFORMACIÓN PARA LA PREVENCIÓN DE DESASTRES TÉCNICAMENTE PREVISIBLES *
}

\author{
The increased risk of Covid 19 in Colombia as a result the state of \\ information for the prevention of technically foreseeable disasters
}

Jorge Eduardo Vásquez Santamaría**

Recepción: 30 de julio de 2020. Aceptación: 14 de agosto de 2020.

DOI: http://dx.doi.org/10.21017/Rev.Repub.2020.v29.a92

\section{RESUMEN}

El COVID-19 representa un incremento del riesgo. A partir de disposiciones jurídicas y políticas se hace preciso contar con elementos para su gestión, entre los cuales están los sistemas de información dispuestos por mandatos internacionales y nacionales. No obstante, su desactualización, desarticulación o inexistencia desencadena un incremento en el riesgo que parece agravarse en aquellos territorios en los que históricamente los sistemas de información llegaron a registrar altas afectaciones como consecuencia de desastres naturales. El trabajo se propone contrastar la funcionalidad de esos sistemas de información para la gestión del riesgo de desastres con las necesidades de la pandemia por COVID-19 en Colombia. Por medio de una investigación cualitativa que aplica la estrategia documental con un análisis de datos respaldado en la triangulación, inicialmente se ofrece una contextualización de la gestión del riesgo de desastres en el campo jurídico-político, se abordan los sistemas de

* Resultado de la investigación «Acceso a la justicia para la protección de derechos colectivos y del ambiente, el acceso a la información y la participación democrática. Fase I Medellín-Acciones Populares», desarrollado en convenio entre la Universidad Católica Luis Amigó desde la línea de investigación «Derecho y Sociedad» y la Universidad Autónoma Latinoamericana desde la línea de investigación «Constitucionalismo Crítico y Género».

** Abogado y Magíster en Derecho de la Universidad de Medellín. Estudiante del Doctorado en Derecho de la Universidad Carlos III de Madrid, España. Docente investigador del Grupo de Investigaciones Jurídicas y Sociales, Facultad de Derecho y Ciencias Políticas, Universidad Católica Luis Amigó, Medellín, Colombia. Orcid: 0000-00026280-005X Google Scholar: https://scholar.google.com.co/citations?user=v8GTVAAAAAJ\&hl=es - jorge.vasquezsa@amigo.edu.co 
información, y finalmente se contrasta la funcionalidad de estos en momentos de pandemia.

Palabras clave: COVID-19, gestión del riesgo de desastres, información.

\begin{abstract}
COVID-19 represents an increased risk. Based on the legal and political provisions, it is necessary to have elements for their mitigation, among them are the information systems provided by international and national legal mandates. However, their lack of updating, disarticulation or nonexistence triggers an increase in risk that seems to be aggravated in those territories where historically the information systems came to register greater damages as a consequence of natural disasters. This work aims to contrast the functionality of these information systems for disaster risk management with the needs of the pandemic in Colombia. Through qualitative research that applies the documentary strategy with a data analysis supported by triangulation, initially a contextualization of disaster risk management in the legal-political field is offered, information systems are addressed, and finally Contrast their functionality in times of a pandemic.
\end{abstract}

Key words: COVID-19, disaster risk management, information systems.

\title{
INTRODUCCIÓN
}

En materia de gestión del riesgo de desastres, Colombia ha seguido la tendencia de suscribir los instrumentos internacionales, y a nivel interno hay evidencias sobre la concreción de sus contenidos. El Estado ha acogido los tres instrumentos principales de ese sector: La Estrategia de Yokohama (1994), posteriormente el Marco de Acción de Hyogo 2005-2015 (2005), y el más reciente fue el Marco de Acción de Sendai para la Reducción de Riesgo de Desastres 2015-2030.

Este último previó «el intercambio abierto y la divulgación de datos desglosados, incluso por sexo, edad y discapacidad, así como de la información sobre los riesgos fácilmente accesible, actualizada, comprensible, con base científica y no confidencial, complementada con los conocimientos tradicionales» (2015, p. 13). Con base en compromisos como los descritos, Colombia desarrolló un marco jurídico que incluye una renovada institucionalidad encargada del diseño y la operación de los sistemas de información. Estos son bases para la debida gestión del riesgo, asumido como la suma de amenazas y vulnerabilidad de la población en el territorio. 
Con la llegada del COVID-19 el incremento en el nivel de riesgo era inminente, en la medida que se suma a problemas de contaminación atmosférica y al incremento de enfermedades respiratorias por el periodo de lluvias. No obstante, la zonificación de territorios vulnerables y la identificación de población en riesgo preexistente por eventos como la contaminación o los desastres naturales, no se constituye en un respaldo para la administración de la pandemia. La desactualización de algunos sistemas de información, su desarticulación o su inexistencia desencadenan un incremento en el riesgo que parece agravarse en aquellos territorios en los que históricamente los sistemas de información existentes llegaron a registrar mayores afectaciones como consecuencia de desastres naturales.

El COVID-19 es un hecho que obliga a la ejecución de acciones administrativas tanto previsibles como precautorias. Previsibles, gracias al conocimiento adquirido que permite actuar con responsabilidad para la protección de los derechos; precautorias, frente a los aspectos en los cuales hay ausencia de la certeza científica para enfrentar el virus, pero que no dejan de ser motivación para la ejecución de acciones. Las falencias identificadas con los sistemas de información se traducen en la pérdida de eficacia, eficiencia y certeza que debe respaldar la capacidad de toma y ejecución de decisiones frente a poblaciones en situaciones de riesgo a las cuales se debe mayor atención y protección por la presencia del COVID-19, lo que en el Estado Social y Democrático de Derecho se debe materializar con acciones afirmativas.

El acceso a la información disponible, que es parcial y desactualizada, permite corroborar la alta vulnerabilidad frente a los fenómenos hidrometeorológicos. Con base en la información existente es posible cruzar esos datos de eventos naturales con la información que refleja la localización del número de contagios en los territorios, y verificar en cuáles de ellos debe existir una atención prioritaria y de máximo nivel preventivo al estar históricamente definidos por el riesgo preexistente de eventos naturales.

\section{METODOLOGÍA}

Se acoge el modelo cualitativo y se aplica la estrategia de investigación documental con un análisis de datos respaldado en la triangulación. La investigación documental se empleó «para realizar la revisión de antecedentes de un objeto de estudio y para reconstruirlo conceptualmente» (Yuni y Urbano, 2014, p. 99), lo que para el caso se focaliza en el referente institucional dedicado a la gestión del riesgo de desastres y la planeación territorial con alcance preventivo frente a comunidades en riesgo. 
Al tiempo, la estrategia documental ofrece la oportunidad de «volver la mirada a un tiempo pasado para comprender e interpretar una realidad actual (sincrónica) a la luz de acontecimientos pasados que han sido los antecedentes que han derivado en los consecuentes de situaciones, acontecimientos y procesos de una realidad determinada» (Yuni y Urbano, 2014, p. 99-100). Lo anterior se materializa con los datos cualitativos que se desprenden de los consolidados del Sistema Nacional de Información -SNI- adscrito al Sistema Nacional de Gestión del Riesgo de Desastres (SNGRD) entre el año 2000 y el 2014. Ese fue el periodo identificado con información disponible, y fue necesario sistematizarlo a partir de las unidades de información acogidas por el mismo SNI, las cuales son insuficientes frente a las previstas por el Marco de Acciones de Sendai.

De lo anterior se desprende que en el análisis de datos, asumido como «el proceso mediante el cual se organiza y manipula la información recogida por los investigadores para establecer relaciones, interpretar, extraer significados y conclusiones» (Spradley, 1980, p. 70), se partiera de un momento de reducción de información a partir de la categoría «Víctimas», desagregadas en las subcategorías muertos, heridos y desaparecidos. La triangulación con el incremento potencial del riesgo a causa del COVID-19 se hizo con las cifras del Instituto Nacional de Salud (INS), empleando para el cruce la localización de población desagregada en entidades territoriales departamentales.

El escrito se estructura a partir de los siguientes componentes: inicialmente se realiza una contextualización de la gestión del riesgo de desastres en el ámbito jurídico-político de Colombia, luego se abordan los sistemas de información, y finalmente se contrasta la funcionalidad de estos en momentos de la pandemia generada con el COVID-19.

\section{LA GESTIÓN DEL RIESGO DE DESASTRES EN EL ÁMBITO JURÍDICO-POLÍTICO DE COLOMBIA}

Los desastres naturales son expresión y resultado de las relaciones históricas y sociales que los colectivos humanos entablan con la naturaleza (Serna, 2011, p. 200), algo en lo que coincide Lavell (1993) que, contrario a un enfoque «fisiquista» que define la anormalidad del evento, propone que los desastres «deben verse más bien como una conformación coyuntural de las condiciones normalmente existentes en la sociedad; de las vulnerabilidades y capacidades humanas preexistentes» (p. 48).

En el ordenamiento jurídico y político de Colombia el desastre es una figura expresamente asociada con el citado enfoque teórico. El desastre tiene una 
relación con el riesgo, y este a su vez depende de la vulnerabilidad y la amenaza. La Ley 1523 de 2012, que regula el SNGRD, se desarrolla a partir de esa relación. En ella el desastre es «el resultado que se desencadena de la manifestación de uno o varios eventos naturales o antropogénicos no intencionales que al encontrar condiciones propicias de vulnerabilidad en las personas, los bienes, la infraestructura, los medios de subsistencia, la prestación de servicios o los recursos ambientales, causa daños o pérdidas humanas (...)» (Ley 1523, 2012, artículo 4.8).

Como resultado que provoca la pérdida y el daño, el desastre requiere de un detonante. Es allí donde la Ley 1523 de 2012 relaciona el riesgo como el evento desencadenante del desastre, en la medida que lo asume como la potencialidad de daño o pérdida ante la ocurrencia del evento (Ley 1523, 2012, artículo 4.25). De manera que conforme a las disposiciones jurídicas el riesgo puede llevar a la ocurrencia del desastre. En ese orden, la amenaza y la vulnerabilidad son los componentes que integran el riesgo del desastre. Ese enfoque, sustentado por White (1974), en el cual el riesgo a sufrir un desastre está asociado a la magnitud de la amenaza natural y a la vulnerabilidad a la que se expone la sociedad (Maskrey, 1993), tiene lugar en las disposiciones jurídicas a partir de las cuales se desarrolla la normativa y se expiden las políticas en la materia.

De un lado, la amenaza es asumida como el «Peligro latente de que un evento físico de origen natural, o causado, o inducido por la acción humana de manera accidental, se presente con una severidad suficiente para causar pérdida de vidas, lesiones u otros impactos (...)» (Ley 1523, 2012, artículo 4.3), y la vulnerabilidad, es la «susceptibilidad o fragilidad física, económica, social, ambiental o institucional que tiene una comunidad de ser afectada o de sufrir efectos adversos en caso de que un evento físico peligroso se presente» (Ley 1523, 2012, artículo 4.27). De lo anterior resulta el riesgo como la conjugación de amenaza y vulnerabilidad, entendiendo que esta última es por medio de la cual se pueden interpretar y traducir «procesos cotidianos de segregación económica y política con una identificación más detallada y puntual de aquellos que estarían expuestos al riesgo en ambientes expuestos a amenazas» (Cannon, 1991).

Con base en figuras como las analizadas, en Colombia la gestión del riesgo de desastres se asume como un «proceso social orientado a la formulación, ejecución, seguimiento y evaluación de políticas, estrategias, planes, programas, regulaciones, instrumentos, medidas y acciones permanentes para el conocimiento y la reducción del riesgo y para el manejo de desastres (...)» (Ley 1523, 2012, artículo 1), comprensión que se corresponde con los avances paradigmáticos que explican la gestión del riesgo de desastres, con el paradig- 
ma de la sostenibilidad ambiental que tributó a su consolidación, y con los instrumentos internacionales que han trasladado las responsabilidades correspondientes al Estado de Colombia como sujeto de Derecho internacional que ha suscrito esos instrumentos.

La correspondencia del proceso de gestión del riesgo de desastres con los sustentos paradigmáticos sobre los cuales se ha construido y ha evolucionado el marco jurídico y político en Colombia, son compatibles con aquellas visiones que asumen la gestión del riesgo de desastres como «Un proceso social complejo, cuyo fin último es la reducción o la previsión y control permanente del riesgo de desastres en la sociedad, en consonancia con, e integrada al logro de pautas de Desarrollo Humano, económico, ambiental y territorial, sostenibles» (Lavell, 2003, p. 30).

En lo que se refiere a su correspondencia con el paradigma de la sostenibilidad, la gestión del riesgo de desastres constituye un escenario regulado en Colombia desde «la década de los años setenta, cuando comenzaron a aparecer normas más completas y articuladas al consenso internacional sobre el medio ambiente, lo que incide en la regulación y reglamentación de acontecimientos o fenómenos directamente vinculados con causas naturales» (Rendón y Vásquez, 2015, p. 53).

Disposiciones de la Declaración de Estocolmo de 1972 correspondientes a la planificación ambiental, como las previstas en los principios 13, 14, 15 y 16, actualmente tienen reflejo en contenidos del artículo 80 de la Constitución Política de 1991. La Declaración de Río de Janeiro de 1992 no solo aseguró la adopción de la sostenibilidad a nivel constitucional (Artículo 80), sino también que ese modelo fuera desagregado en las disposiciones de la Ley 99 de 1993 (Vásquez, Gómez y Martínez, 2017). En esa normativa la gestión del riesgo de desastres es un principio que orienta la política nacional ambiental, es función de las Corporaciones Autónomas Regionales, pero además permitió la ampliación del uso y el alcance del principio de precaución (Rendón y Vásquez, 2015, p. 54), el cual, en la actualidad, no solo está previsto para asuntos ambientales (Ley 99, 1993, artículo 1), sino que también es principio del SNGRD (Ley 1523, 2012, artículo 2).

Finalmente, el desarrollo de la gestión del riesgo de desastres en el ordenamiento jurídico y político de Colombia se corresponde con la suscripción de los instrumentos internacionales que han gobernado la materia. La Estrategia de Yokohama -EY- (1994), el Marco de Acción de Hyogo -MAH- 2005-2015 (2005), y el Marco de Acción de Sendai para la Reducción de Riesgo de Desastres 2015-2030, instrumentos que, entre sus disposiciones, hacen explícita la obligación de diseñar e implementar sistemas de información, una garantía de base por medio de la cual se realiza el derecho de acceso a la información. 


\section{LOS SISTEMAS DE INFORMACIÓN Y EL ACCESO A LA INFOR- MACIÓN EN TIEMPOS DE COVID-19}

En la EY la información fue objeto de uno de los principios rectores para adelantar una adecuada gestión del riesgo de desastres, apoyada en su efectiva difusión «mediante las telecomunicaciones, inclusive los servicios de radiodifusión, son factores clave para prevenir con éxito los desastres y prepararse bien para ellos» (1994, principio 5). También fue prevista como parte de las estrategias para el año 2000 y contenido de las recomendaciones a nivel local y comunitario, primordialmente como fuente de concienciación pública, tendencia que se siguió en el plano regional y nacional.

En el MAH 2005-2015 (Conferencia Mundial sobre Reducción de los Desastres, 2005) aumentó el peso de su incidencia. La información fue prevista como uno de los contenidos de los objetivos generales (2005, p. 3), una de las prioridades de acción (2005, p. 5), pero sobre todo, como una de las actividades esenciales para materializar la acción prioritaria enfocada en «Utilizar los conocimientos, las innovaciones y la educación para crear una cultura de seguridad y de resiliencia a todo nivel» $(2005$, p. 9).

Su mayor grado de especialización y transversalización en la gestión del riesgo de desastres llega con el Marco de Acción de Sendai. En él, se incluye como principio el abierto intercambio y divulgación de datos «desglosados, incluso por sexo, edad y discapacidad, así como de la información sobre los riesgos fácilmente accesible, actualizada, comprensible, con base científica y no confidencial, complementada con los conocimientos tradicionales» (Tercera Conferencia Mundial sobre Reducción de los Desastres, 2015, p. 13). Prevé el deber de los Estados de «Promover el acceso en tiempo real a datos fiables, hacer uso de información espacial e in situ, incluidos los sistemas de información geográfica (SIG), y utilizar las innovaciones en materia de tecnología de la información y las comunicaciones para mejorar los instrumentos de medición y la recopilación, el análisis y la difusión de datos» (2015, p. 15). La recopilación, el análisis, la gestión y el empleo de la información son algunas de las acciones para alcanzar la comprensión del riesgo de desastres, al mismo tiempo que lo es el deber de elaborarla, actualizarla y difundirla con base en cartografías y mapas (2015, p. 15), o «Asegurar que la información no confidencial desglosada por pérdidas sobre el grado de exposición a amenazas, la vulnerabilidad, los riesgos y los desastres esté disponible y accesible libremente, como corresponda» (2015, p. 15).

Con base en ese marco de referencia es preciso abordar dos interrogantes: ¿Cómo se materializan las obligaciones estatales sobre la información en gestión del riesgo de desastres? y ¿Qué función cumplen ante una pandemia como 
el COVID-19? Para dar respuesta a estas preguntas no se debe perder de vista que en Colombia la gestión del riesgo de desastres articula el conocimiento y la reducción del riesgo, y el manejo de los desastres.

El primero se asume como «la identificación de escenarios de riesgo, el análisis y evaluación del riesgo, el monitoreo y seguimiento del riesgo y sus componentes y la comunicación para promover una mayor conciencia del mismo» (Ley 1523, 2012, artículo 4.7). Desde ese alcance la información tiene un rol de base, toda vez que la ejecución de las acciones que implica el conocimiento del riesgo parte del efectivo acceso a la información, de su divulgación y su apropiación, pero sobre todo de su existencia y elaboración, para lo cual resultan imperiosos los términos establecidos en los instrumentos internacionales que incorporan la información en la gestión del riesgo de desastres. Además, no puede pasar como un dato aislado que en Colombia el manejo y la transferencia de la información, así como el diseño y la operatividad del SNI, son funciones que se encomiendan al Comité Nacional para el Conocimiento del Riesgo, instancia encargada de las acciones de base de las que depende la reducción del riesgo, y el manejo de desastres.

En lo que respecta a la reducción del riesgo, la Ley 1523 de 2012 establece que conlleva el proceso de gestión «compuesto por la intervención dirigida a modificar o disminuir las condiciones de riesgo existentes, entiéndase: mitigación del riesgo y a evitar nuevo riesgo en el territorio, entiéndase: prevención del riesgo» (Artículo 4.21). Con ello, el acceso a la información que sirve de presupuesto al conocimiento de base es igualmente condición necesaria para poder emprender la mitigación y la prevención que dan lugar a la reducción del riesgo. Solo con la construcción, la divulgación y el acceso debido a la información será posible emprender la definición de acciones de mitigación y prevención en casos concretos en los que se pueda definir la amenaza y la vulnerabilidad a la cual está sometida una población en un territorio.

En la misma dirección se enlaza la relación información-manejo de desastres. Apoyado en el conocimiento y la reducción del riesgo, el manejo de desastres conlleva «la preparación para la respuesta a emergencias, la preparación para la recuperación posdesastre, la ejecución de dicha respuesta y la ejecución de la respectiva recuperación, entiéndase: rehabilitación y recuperación» (Ley 1523, 2012, artículo 4.15). Todas las acciones previstas como parte de la gestión del manejo del desastres acarrean el acceso a la información como condición de base para su efectiva ejecución.

De esa manera se desprende que el acceso a la información cumple varios roles dentro del proceso de gestión del riesgo de desastres: inicialmente, y parece redundante, la información incorpora su dimensión de derecho de acceso pre- 
visto en el principio 10 de la Declaración de Río de Janeiro de 1992; es un componente formal y material que transversaliza la operatividad del SNGRD; es un componente en el que se reflejan los contenidos de los instrumentos internacionales sobre la materia como pautas orientadoras e interpretativas, cuando no procedan como disposiciones con un carácter vinculante; y se trata de una dimensión ius fundamental del derecho a la prevención de desastres técnicamente previsibles, en el sentido que le aporta los contenidos de un derecho constitucional esencial como es la información, lo que abre paso a la interdependencia y la conexidad entre derechos.

De ello que la Ley 1523 respalde al SNGRD en el principio de oportuna información. A través de él las autoridades tienen el deber de «mantener debidamente informadas a todas las personas naturales y jurídicas sobre: Posibilidades de riesgo, gestión de desastres, acciones de rehabilitación y construcción así como también sobre las donaciones recibidas, las donaciones administradas y las donaciones entregadas» (Artículo 3, 15). La información está además prevista como uno de los insumos que hace posible la preparación y la respuesta (Artículo 4.17-4) y es componente del SNGRD (Artículo 5), se fija como uno de sus objetivos (Artículo 6.e), y por ello, hace que en la Ley se reconozcan los sistemas de información como uno de los componentes principales delSNGRD (Artículo 7.3). La Ley 1523 impone la operatividad del SNI, en el cual deben confluir los contenidos y datos de todas las entidades nacionales y territoriales al ser el insumo para el conocimiento y reducción del riesgo, y para el manejo de los desastres. El SNI se respalda en los sistemas de información de los niveles territoriales, con los cuales debe asegurar una interoperabilidad (Artículo 46).

A partir de lo anterior se evidencia que en el ordenamiento jurídico y político de Colombia se han dispuesto los lineamientos por medio de los cuales el Estado y la sociedad deben emprender la gestión del riesgo de desastres asegurando la incorporación de lo que denominaremos «variable informativa». Se trata de un componente para la planificación territorial que es «un verdadero sistema de gobierno corporativo [que] consiste en el manejo y tratamiento de aquellas situaciones que siendo inciertas en cuanto a su ocurrencia y/o impacto, tienen un efecto en el cumplimiento de los objetivos trazados por la organización» (Safar, 2012, p. 46), y donde su ausencia «constituye una fuente de ineficiencia en la administración pública que deriva en la configuración de una responsabilidad por la eventual causación de daños antijurídicos generada en la omisión de los órganos directamente llamados a su realización» (Safar, 2012, p. 47).

Agotadas algunas reflexiones sobre el primer interrogante, es preciso emprender la elaboración de una respuesta frente al segundo de ellos: ¿Qué función 
cumplen los sistemas de información ante una pandemia como el COVID-19? La respuesta se puede dividir en varios componentes. El primero nos obliga a reiterar que estamos en presencia de un derecho constitucional de tipo fundamental que en el escenario ambiental que sirvió de nicho a la gestión del riesgo de desastres, conlleva el componente de «acceso». De manera que a la luz del principio 10 de la Declaración de Río de Janeiro de 1992, el acceso a la información es uno de los componentes que conducen al «mejor modo de tratar las cuestiones ambientales», y adicional, es una potestad esencial del individuo que se puede ver comprometida en la gestión del riesgo de desastres.

El ejercicio de ese contenido ius fundamental cumple funciones garantistas en el SNGRD. Contribuye a la protección de otros derechos fundamentales, como la vida, la salud, la integridad física, la dignidad, la participación o la justicia, y otros de tipo colectivo, como el ambiente sano, el equilibrio ecológico, la construcción conforme a la normativa urbanística, o la seguridad.

Las funciones garantistas de la información resultan obligatorias ante un evento como el COVID-19. La Ley 1523 de 2012 incluye dentro de las posibilidades de desastre los eventos antropogénicos no intencionales que al encontrar condiciones adecuadas causan daños o pérdidas (Artículo 4.8), algo que se puede constatar con el COVID-19. No se trata de un evento antropogénico intencionado, y en su propagación ha puesto en evidencia las profundas condiciones de vulnerabilidad de los seres humanos, de sus bienes, infraestructuras y medios de subsistencia sin distingo de clase. Se confirma la pandemia del COVID-19 como objeto de tratamiento por parte del SNGRD, que se reitera en la modalidad de riesgo, que incluye «eventos físicos peligrosos de origen natural, socio-natural tecnológico, biosanitario o humano no intencional» (Artículo 4.25).

De manera que en Colombia el Comité Nacional para el Conocimiento del Riesgo (Ley 1523, 2012, artículo 20), como encargado legal de «Formular lineamientos para el manejo y transferencia de información y para el diseño y operación del Sistema Nacional de Información para la Gestión del Riesgo» (Ley 1523, 2012, artículo 21), ha estado en la obligación de proporcionar derroteros por medio de los cuales el SNI recibe, sistematiza y permite la divulgación de la información de la pandemia para la concienciación pública y la operatividad interinstitucional. Además, dichos lineamientos deben ser los articuladores que den lugar a la interoperabilidad con los sistemas de las entidades territoriales; pero más aún, son los que permiten identificar, localizar y comprender las condiciones de vulnerabilidad de la población en el territorio.

No hacemos referencia a las condiciones de vulnerabilidad frente a la pandemia, sino a las condiciones de vulnerabilidad preexistente, de carácter preventivo, 
entendidas como la «Susceptibilidad o fragilidad física, económica, social, ambiental o institucional que tiene una comunidad de ser afectada o de sufrir efectos adversos» (Ley 1523, 2012, artículo 4.27). El SNI, previo a la ocurrencia del COVID-19, concentraba el cumplimiento de la obligación a través de la cual las autoridades estatales y la sociedad debían comprender la localización, la caracterización y el estado de comunidades vulnerables por eventos diferentes a la pandemia, pero que frente a la llegada del COVID-19, presentan un incremento en el nivel de amenaza y vulnerabilidad, esto es del riesgo, y por ello, del daño y la pérdida.

\section{DISCUSIÓN}

La pandemia del COVID-19 pone a la sociedad global en una situación crítica en la cual se incrementan las condiciones de vulnerabilidad: «Las consecuencias de este desastre pudieran cambiar, por mucho tiempo, las relaciones económicas, sociales e interpersonales en el mundo y cada uno de nuestros países» (UNESCO, 2020). En la misma tendencia se pronunció la Oficina de las Naciones Unidas para la Reducción de Desastres (UNDRR, por sus siglas en inglés):

aceptar una nueva comprensión sobre la naturaleza dinámica de los riesgos sistémicos, establecer nuevas estructuras para gestionarlo en sistemas complejos y adaptativos, y elaborar una serie de herramientas nuevas para realizar inversiones y tomar decisiones orientadas por el riesgo, lo cual permitiría que las sociedades humanas vivan con y en la incertidumbre (Gordon y Williams, 2020).

En ese panorama es que las relaciones sociales, y en especial las económicas, se están viendo sometidas a transformaciones profundas. Las primeras debido al aislamiento y los cambios en la cotidianidad, lo que conlleva la alteración y la reducción en la comunicación y el acceso a la información que permita establecer certezas frente a las circunstancias del COVID-19, y sobre la evolución de las condiciones que rigen las amplias y abundantes medidas excepcionales adoptadas por el Estado. Las segundas, debido al incremento de la incertidumbre y los bruscos altibajos de la economía que alteran las formas de vida de las comunidades.

Aquellos sectores de la sociedad que ya contaban con condiciones de vulnerabilidad por la existencia de riesgos naturales, en muchas ocasiones ven incrementado su nivel de fragilidad física, económica, social o ambiental por la posible ocurrencia del evento natural que aqueje el territorio donde se localizan. Esto se da porque en tiempos de pandemia la locomoción y el ejercicio de reunión son excepcionales, ejercer la participación es algo que puede llegar a 
ser inviable, y la posibilidad de adquirir el COVID-19 representa una amenaza creciente para la salud y la vida.

Las condiciones preexistentes de vulnerabilidad también aumentan cuando se piensa en el potencial del deterioro de la salud física y mental y de las condiciones de vida debido a los cambios económicos provocados por el COVID-19. Esas condiciones alteran las posibilidades de la población para hacer frente a un desastre, poniendo en un nivel de riesgo la resiliencia que se venía instalando en la gestión del riesgo de desastres previo a la pandemia a partir de las orientaciones fijadas por los instrumentos internacionales.

El COVID-19 no excluye, suspende ni aminora la ocurrencia de los eventos naturales, los cuales hacen parte de la forma de vida de amplios sectores de la población y determinan su forma de ser y de estar en el territorio. La permanencia de poblaciones en zonas vulnerables a eventos naturales concurre con la posibilidad de propagación del COVID-19, lo que representa un riesgo adicional para muchas comunidades, debido a las condiciones de vulnerabilidad y exposición a la amenaza por la ocurrencia del evento natural, a la propensión del contagio, y a la disponibilidad de recursos y de infraestructura habilitada por el Estado para enfrentar el desastre.

De lo anterior que el riesgo por el acaecimiento de desastres a raíz de un evento natural exige la actualización, la vigencia y la operatividad de los sistemas de información de gestión del riesgo de desastres de todos los niveles administrativos del Estado. Los sistemas de información deben ser fuentes efectivas de acceso a la información que permita asegurar el acceso a la localización y caracterización de la población, a la información sobre las amenazas y la vulnerabilidad que los definen en el territorio, a una información accesible, actualizada, comprensible, con base científica y no confidencial, que permita una adecuada planeación y prevención, que pueda priorizar acciones afirmativas sobre grupos humanos en los que se incrementan los factores de riesgo por desastre, emergencia y calamidad, en concurrencia con la propagación del COVID-19.

El acceso a la información que deben proveer los sistemas de información de la gestión del riesgo de desastres debe asegurar el conocimiento del riesgo en tiempos de incremento exponencial de la vulnerabilidad de la población en los territorios. El conocimiento del riesgo asegura la funcionalidad del «proceso de la gestión del riesgo compuesto por la identificación de escenarios de riesgo, el análisis y evaluación del riesgo, el monitoreo y seguimiento del riesgo y sus componentes y, la comunicación para promover una mayor conciencia del mismo que alimenta los procesos de reducción del riesgo y de manejo de desastre» (Ley 1523, 2012, artículo 4.7). 


\section{Funcionalidad de los sistemas de información y del acceso a la información en tiempos de COVID-19}

De cara a la realidad que enfrenta el SNGRD con la expansión del COVID-19 en Colombia, la funcionalidad de los sistemas de información que están llamados a respaldar la ejecución de las acciones que implica el riesgo y el desastre, exige contrastar esa funcionalidad con las necesidades de la pandemia por COVID-19 en el país.

La Unidad Nacional de Gestión del Riesgo de Desastres -UNGRD- es la encargada de «poner en marcha, un sistema nacional de información para la gestión del riesgo de desastres, el cual debe mantenerse actualizado y funcional mediante la integración de contenidos de todas las entidades nacionales y territoriales, (...)» (Ley 1523, 2012, artículo 45). En el año 2014, a dos años de la entrada en vigencia del SNGRD, la UNGRD entonces identificó que en Colombia «No se cuenta con un instrumento a nivel nacional que permita integrar, consolidar y diseminar la información relacionada con gestión del riesgo entre los diferentes actores de la gestión del riesgo de desastres» (2014, p. 11), lo cual asoció a la «Deficiencia de políticas, procedimientos y acuerdos para el manejo de la información relacionada con la gestión del riesgo a nivel nacional» $(2014$, p. 11).

La UNGRD se propuso «Implementar el Sistema Nacional de Información para la Gestión del Riesgo de Desastres, que permita la articulación e integración de la información generada por las entidades de orden nacional y territorial, así como el fomento de su buen uso y generación» (2014, p. 12). Para ello se trazó un desarrollo en etapas, que inició por el sistema de información de base, seguido del sistema de información científica, el sistema de información para emergencias y el sistema de información para la planeación del territorio, y finaliza con el sistema de seguimiento y control (2014).

No obstante, en la actualidad, el SNI no cuenta con registros públicos sobre la forma como contribuye con la adopción, adaptación y promoción de estándares, protocolos, soluciones tecnológicas y procesos para el manejo de información para la gestión del riesgo de desastres. Tampoco se evidencia información pública que permita asegurar el seguimiento de las amenazas, las vulnerabilidades y los riesgos en los territorios. La UNGRD tiene los consolidados de atención a emergencias con información no confidencial disponible públicamente correspondiente a los años 1998 a 2012, pero no dispone de la relacionada con los años 2013, 2014, 2015 y 2016, y si bien reseña los años 2017 y 2018, en la página web de la UNGRD no se despliega información de ninguno de esos dos periodos. 
La UNGRD administra el consolidado de emergencias como parte del SNI. Este está compuesto por la descripción y ubicación del evento natural, la afectación en muertos, heridos, desaparecidos, personas y familias, y el apoyo nacional proporcionado. No obstante, el SNI adolece de la aplicación de información desagregada "por sexo, edad y discapacidad, así como de la información sobre los riesgos fácilmente accesible, actualizada, comprensible, con base científica y no confidencial, complementada con los conocimientos tradicionales» (2015, p. 13), tal como lo establece el Marco de Acción de Sendai.

Unido a las ausencias y debilidades que presenta la funcionalidad del SNI, se identifica que tampoco ha implementado un enfoque diferencial que permita identificar, caracterizar y comprender las condiciones de la población en situación de riesgo y desastre, componente de relevancia si se tiene presente que:

La adopción de medidas a favor de los grupos marginados no constituye una competencia meramente facultativa del legislador, sino que es un mandato de acción, encaminado a transformar las condiciones materiales que engendran o perpetúan la exclusión y la injusticia social. Este deber estatal, si bien necesita ser desarrollado por la ley, y está atado a las apropiaciones presupuestales correspondientes, no puede quedar indefinidamente aplazado en la agenda estatal (Corte Constitucional, 2004, T-025).

En la actualidad, el SNI carece de enfoque étnico, de género, de edad, de discapacidad, de desplazamiento, de composición del grupo familiar, de titularidad sobre la propiedad, pero más aun, de contagios por COVID-19, de condiciones de vulnerabilidad que conlleven potenciales contagios. El SNI no reunió ni reúne datos sobre personas o familias que son objeto de procedimientos de reubicación y reasentamiento a causa de eventos naturales que pueden detonar desastres. A lo anterior se suma que la legislación vigente no dispone de normativa que fije lineamientos o mecanismos específicos para emprender procesos de reubicación y reasentamiento producidos por eventos naturales, pero tampoco alguno que conlleve emprender procesos de reasentamiento y reubicación por eventos antropogénicos no intencionales, como el COVID-19.

El SNI no evidencia las formas, canales, mecanismos o procedimientos por medio de los cuales asegure la operatividad con los sistemas de información regional, departamental, municipal y distrital. Lo anterior define la funcionalidad del SNI a partir de parámetros de poca eficacia, eficiencia, transparencia y responsabilidad administrativa. 


\section{A MODO DE CONCLUSIÓN}

En Colombia el COVID-19 se traduce en un evento con características que exigen la ejecución de acciones administrativas de tipo preventivo y precautorio, dando aplicación a los contenidos del principio de responsabilidad y de precaución que rige el SNGRD. Las primeras, gracias al conocimiento adquirido que permite actuar para proteger los derechos de la población vulnerable, tales como las medidas de aislamiento, confinamiento, suspensión de actividades, ejecución de medidas de higiene para garantizar la salud pública, todas respaldadas en el acceso a la información científica. Precautorias, frente a los aspectos en los cuales hay ausencia de la certeza científica para enfrentar el virus, pero que no por eso dejan de ser motivación para la ejecución de acciones. Estas últimas son las que de manera particular presentan una demanda aún mayor de la información que deben proveer los sistemas, en la medida que la ausencia de certeza científica que permite la ejecución de acciones mucho más restrictivas, requiere una información de base que permita establecer con seguridad la existencia de ausencia de certeza científica.

Las falencias del SNI se traducen en la pérdida de eficacia, eficiencia y certeza que deben respaldar la capacidad de toma y ejecución de decisiones frente a poblaciones en situaciones de riesgo por eventos naturales a las cuales se debe proveer una mayor y prioritaria atención y protección ante el COVID-19. En la experiencia colombiana un ejemplo en el que concurren todas las debilidades que se desprenden de los sistemas de información y del acceso a la información sobre el evento se evidencia con la situación de comunidades indígenas de la Amazonía, donde el nivel de daños y pérdidas supera por mucho las posibilidades de prevención y mitigación.

Con base en esa información, aunque parcial (UNGRD, 2020), es posible cruzar los datos de riesgos con la información que refleja el número de contagios en los territorios (INS, 2020), lo que arroja un panorama preocupante, como indicio de la concurrencia de altos niveles de contagio en lugares con alta concentración demográfica y propensión a la ocurrencia de desastres por eventos naturales. Tablas 1, 2 y 3.

En el caso del acceso a la información relacionada con la localización, caracterización e identificación de las personas contagiadas por COVID-19 en Colombia, se identifica un sistema de información dispuesto, gestionado y actualizado por el INS que ofrece información por casos, departamento, municipio, población, muestras y remisión a otras fuentes de información sobre la pandemia. En lo que corresponde a la información por municipio, el sistema de información del INS da cuenta de los casos confirmados, recuperados y fallecidos, así como del sexo y la edad de los contagiados, la atención prestada y el tipo de 

información para la prevención de desastres técnicamente previsibles

Tabla 1. Número de eventos hidrometeorológicos en los departamentos con mayor densidad demográfica en Colombia 2011-2014

\begin{tabular}{|l|c|c|c|c|c|c|c|c|c|c|c|c|}
\hline & \multicolumn{4}{|c|}{ Inundación } & \multicolumn{5}{c|}{ Deslizamiento } & \multicolumn{3}{c|}{ Vendaval } \\
\hline Departamento & $\mathbf{2 0 1 1}$ & $\mathbf{2 0 1 2}$ & $\mathbf{2 0 1 3}$ & $\mathbf{2 0 1 4}$ & $\mathbf{2 0 1 1}$ & $\mathbf{2 0 1 2}$ & $\mathbf{2 0 1 3}$ & $\mathbf{2 0 1 4}$ & $\mathbf{2 0 1 1}$ & $\mathbf{2 0 1 2}$ & $\mathbf{2 0 1 3}$ & $\mathbf{2 0 1 4}$ \\
\hline Antioquia & 160 & 10 & 58 & 27 & 44 & 26 & 38 & 19 & 0 & 30 & 21 & 177 \\
\hline Atlántico & 56 & 3 & 22 & 6 & 10 & 1 & 1 & 1 & 0 & 0 & 43 & 74 \\
\hline Bolívar & 72 & 1 & 12 & 15 & 8 & 8 & 20 & 7 & 0 & 16 & 17 & 74 \\
\hline Cundinamarca & 242 & 4 & 49 & 36 & 93 & 48 & 41 & 44 & 122 & 29 & 43 & 22 \\
\hline Santander & 90 & 10 & 28 & 21 & 5 & 63 & 50 & 40 & 9 & 44 & 25 & 15 \\
\hline Valle del Cauca & 122 & 12 & 59 & 26 & 26 & 13 & 21 & 11 & 17 & 22 & 45 & 30 \\
\hline
\end{tabular}

Elaboración propia con base en los datos de Gómez, M. I., Martínez, H. D., \& Vásquez, J. E. (2018). Gestión del riesgo en Colombia. Derechos Humanos en procesos de reasentamiento de población vulnerable. Medellín: Universidad Autónoma Latinoamericana.

Tabla 2. Número de personas contagiadas por COVID-19 en los departamentos con mayor densidad demográfica en Colombia

\begin{tabular}{|l|c|c|c|c|c|}
\hline \multicolumn{1}{|c|}{ Departamento } & 23 Mayo & 23 Julio & 23 Agosto & 23 Septiembre & 23 Octubre \\
\hline Antioquia & 886 & 23697 & 74350 & 111025 & 156306 \\
\hline Atlántico & 2330 & 48947 & 63291 & 67163 & 70746 \\
\hline Bolívar & 851 & 17098 & 24398 & 29116 & 32153 \\
\hline Cundinamarca & 35088 & 86732 & 215380 & 294844 & 348850 \\
\hline Santander & 85 & 2633 & 14870 & 29765 & 39928 \\
\hline Valle del Cauca & 1276 & 19217 & 41567 & 58048 & 75734 \\
\hline
\end{tabular}

Elaboración propia con base en los datos del Instituto Nacional de Salud de la República de Colombia (2020). https://www.ins.gov.co/Noticias/Paginas/Coronavirus.aspx

importación, además de valerse de información georreferenciada ofrecida a partir de cartografía actualizada de forma reciente. Por departamento, el INS asegura el acceso a la información a través de consolidados de casos discriminados por sexo y edad, y especificando la situación de los casos en número de nuevos contagios, recuperados, fallecidos y hospitalizados.

El sistema de información empleado por el INS sigue la organización escalar administrativa que está dispuesta por la Ley 1523 de 2012 para la creación de sistemas de información en las entidades territoriales, esto es, una escala que trabaja a partir de los consolidados generados por municipios, distritos y de- 
Tabla 3. Número de personas fallecidas por COVID-19 en los departamentos con mayor densidad demográfica en Colombia

\begin{tabular}{|l|c|c|c|c|c|}
\hline \multicolumn{1}{|c|}{ Departamento } & 23 Mayo & 23 Julio & 23 Agosto & 23 Septiembre & 23 Octubre \\
\hline Antioquia & 19 & 541 & 1851 & 2565 & 3042 \\
\hline Atlántico & 137 & 2635 & 2972 & 3081 & 3117 \\
\hline Bolivar & 47 & 608 & 722 & 792 & 833 \\
\hline Cundinamarca & 173 & 3057 & 6424 & 8003 & 8685 \\
\hline Santander & 4 & 118 & 839 & 1392 & 1622 \\
\hline Valle del Cauca & 91 & 868 & 1701 & 2180 & 2543 \\
\hline
\end{tabular}

Elaboración propia con base en los datos del Instituto Nacional de Salud de la República de Colombia (2020). https://www.ins.gov.co/Noticias/Paginas/Coronavirus.aspx

partamentos. Si bien no se identifica la aplicación de los enfoques diferenciales y tampoco una desagregación de condiciones de vulnerabilidad como integración del núcleo familiar, estratificación socioeconómica, o titularidad de pólizas de salud, en comparación con el SNI, el acceso que permite el INS es mayor en términos de celeridad, eficacia y eficiencia.

\section{REFERENCIAS}

Cannon, T. (1991). «A Hazard Need Not a Disaster Make: Rural Vulnerability and the Causes of Natural Disaster». Ponencia presentada en la Conferencia: Disasters: Vulnerability and Response, DARG/IBG/RGS, London.

Conferencia Mundial sobre Reducción de los Desastres (2005). Marco de Acción de Hyogo para 2005-2015: Aumento de la Resiliencia de las naciones y las comunidades ante los desastres. Disponible en https:/ / www.eird.org/cdmah/contenido/ hyogo-framework-spanish.pdf

Conferencia Mundial sobre Reducción de los Desastres, la preparación para casos de desastre y la mitigación de sus efectos (1994). Estrategia y Plan de Acción de Yokohama para un mundo más seguro. Disponible en https:/ / eird.org/fulltext/ Yokohama-strategy/YokohamaEspa\%F1ol.pdf

Congreso de la República de Colombia (1993). Ley 99.

Congreso de la República de Colombia (2012). Ley 1523.

Gómez Vélez, M. I., Martínez Hincapié, H. D., \& Vásquez Santamaría, J. E. (2018). Gestión del riesgo en Colombia. Derechos Humanos en procesos de reasentamiento de población vulnerable. Medellín: Universidad Autónoma Latinoamericana. 
Gordon, M. \& Williams, S. (2020). ¿Por qué la compresión de la naturaleza sistémica del riesgo es muy importante en medio de la crisis del COVID-19? Disponible en https:/ / eird.org/americas/noticias/por-que-la-comprension-de-la-naturalezasistemica-del-riesgo-es-muy-importante-en-medio-de-la-crisis-del-COVID-19.html

Instituto Nacional de Salud de la República de Colombia (2020). COVID-19 en Colombia. Disponible en: https://www.ins.gov.co/Noticias/Paginas/Coronavirus.aspx

Lavell, A. (1993). Comunidades urbanas, vulnerabilidad a desastres y opciones de prevención y mitigación: Una propuesta de investigación-acción para Centroamérica. En: Viviendo en Riesgo. Comunidades vulnerables y prevención de desastres en América Latina. Lima: Red de Estudios Sociales en Prevención de Desastres en América Latina.

Lavell, A. (2003). Reducción del riesgo de desastres en el ámbito local: lecciones desde la subregión andina. Lima, Perú: Secretaría General de la Comunidad Andina. Recuperado de http://www.desenredando.org/public/varios/2009/2009_ PREDECAN_Lavell_Gestion\%20Local\%20del\%20Riesgo.pdf

Maskrey, A. (1993). Comunidades y desastres en América Latina: Estrategias de Intervención. En: Viviendo en Riesgo. Comunidades vulnerables y prevención de desastres en América Latina. Lima: Red de Estudios Sociales en Prevención de Desastres en América Latina.

Rendón Osorio, K. V., y Vásquez Santamaría, J. E. (2015). Reconstrucción normativa sobre gestión del riesgo 1970-1989: componente clínico para un informe de ampliación a la CIDH sobre derechos humanos en poblaciones propensas a procesos de reasentamiento. Indisciplinas, 1(2), 45-73.

Safar Díaz, M. S. (2012). Responsabilidad de las entidades territoriales en la gestión del riesgo como política pública. Revista Digital de Derecho Administrativo, (7), 45-62.

Serna Quintana, C. A. (2011). La naturaleza de los desastres asociados a inundaciones y deslizamientos en Medellín. Historia Crítica, (43), 198-223.

Spradley, J. P. (1980). Qualitative Analysiss for Social Scientists. Cambridge: University Press.

Tercera Conferencia Mundial sobre Reducción de los Desastres (2015). Marco de Sendai para la reducción del Riesgo de Desastres 2015-2030. Disponible en: https:// www.unisdr.org/files/43291_spanishsendaiframeworkfordisasterri.pdf

UNESCO (2020). El riesgo de desastres de origen natural en tiempos de Covid-19. Disponible en: https:/ / es.unesco.org/news/riesgo-desastres-origen-natural-tiempos-covid-19 
Unidad Nacional para la Gestión del Riesgo de Desastres -UNGRD- (2014). Implementación del Sistema Nacional de Información para la gestión del riesgo de desastres 2015-2018. Bogotá: Unidad Nacional para la Gestión del Riesgo de Desastres UNGRD-.

Unidad Nacional para la Gestión del Riesgo de Desastres -UNGRD- (2020). Sistema Nacional de Información para la Gestión del Riesgo de Desastres. Disponible en: http://gestiondelriesgo.gov.co/snigrd/emergencia.aspx?id=41

Vásquez Santamaría, J. E.; Gómez Vélez, M. I.; Martínez Hincapié, H. D. (2017). Gestión del riesgo de desastres, ordenamiento territorial, reasentamiento y reubicación: correlación desequilibrada para el ejercicio de derechos humanos desde retrospectivas jurídicas en Colombia. En: Seminario Internacional de Investigación en Urbanismo. «IX Seminario Internacional de Investigación en Urbanismo, Barcelona-Bogotá, junio 2017». Barcelona: DUOT.

White, G. (1974). Natural Hazards: Local, National, Global. New York: Oxford University Press.

Yuni, J. y Urbano, C. (2014). Técnicas para investigar II. Recursos metodológicos para la preparación de proyectos de investigación. Córdoba: Editorial Brujas. 
